\title{
Challenging the Myth: Directional Atherectomy-A Reply
}

\author{
Dierk Vorwerk
}

Received: 7 November 2008/Accepted: 7 November 2008/Published online: 11 December 2008

(C) Springer Science+Business Media, LLC 2008

"Challenging the myth" is a new column we have introduced to stimulate discussions among our readers to give their ideas and thoughts about controversial principles and issues. The content of these short commentaries will be influenced by individual and sometimes subjective thoughts and conclusions. However, these opinions should not be published without the opportunity for others to give an alternative view if they so wish. Therefore, we also intend to create space in the journal where authors can provide opposing viewpoints.

Jim Reekers starts this series by challenging the myth of atherectomy and points out that there was never any proof that atherectomy works better than balloon angioplasty, neither in cohort series nor in randomized trials, and I have to agree that he is right.

Nevertheless, we need atherectomy. Many interventional radiologists missed the old Simpson atherectomy catheter when it disappeared because there was no better tool for debulking eccentric calcified plaques, especially from the popliteal artery in the P2 segment. Therefore, despite the fact that no statistical benefit was proven over PTA, atherectomy is a valuable and helpful tool although it is not required on a frequent basis.

Clearly, this should be the approach regarding how we should view the new atherectomy device, i.e., as a tool and not as miraculous new weapon. It might be a mistake to advertise the device as something revolutionary that will drastically change long-term results in peripheral angioplasty. However, the new atherectomy device is a helpful

D. Vorwerk $(\bowtie)$

Department of Radiology, Klinikum Ingolstadt, Krumenauerstrasse 25, Ingolstadt 85049, Germany e-mail: dierk.vorwerk@klinikum-ingolstadt.de new instrument that is much more versatile than the old Simpson device.

The new atherectomy device is useful to remove organized as well as fresh thrombus. The device works on calcified material (although not as well as the Simpson device), and it can debulk eccentric plaques from small arteries without a significant risk of dissection. It is also useful to remove dissections after balloon angioplasty, which otherwise require stent implantation. It can be used on tight and fibrous lesions, such as anastomotic strictures in bypasses, and helps to clean restenosed stents by removing neointimal tissue (not officially, but I swear it works). It is therefore a useful tool especially in delicate areas, such as close to the groin or in the popliteal artery.

Professor Reekers is correct in that there is no proof that the device creates better follow-up results than angioplasty alone, although in the registry there was some interest in the fact that diabetic patients had improved patency compared with diabetic patients treated with alternative techniques. However, all of this remains to be proven in a randomized trial.

I know that Professor Reekers loves cooking to a great degree. He would love to have such a new multifunctional tool in his kitchen to help him out in technically challenging situations. This is exactly the way I am looking at this new device. If it proves to be better than angioplasty, that's great. If it fails, I would not be surprised, but I would still like the device as a valuable tool in particular acute situations.

Professor Reekers says myths in interventional radiology sometimes are challenged twice. However, he forgets the example of femoral stenting, which was abandoned once but has definitely changed our approach to femoral disease in a second run after the introduction of nitinol stents. 